\title{
Atrioventricular Block First Degree, CTCAE
}

National Cancer Institute

\section{Source}

National Cancer Institute. Atrioventricular Block First Degree, CTCAE. NCI Thesaurus.

Code C143309.

A disorder characterized by a dysrhythmia with a delay in the time required for the

conduction of an electrical impulse through the atrioventricular (AV) node beyond 0.2 seconds; prolong ation of the PR interval greater than 200 milliseconds. 